\title{
Self-Organization and Artificial Life: A Review
}

\author{
Carlos Gershenson ${ }^{1}$, Vito Trianni ${ }^{2}$, Justin Werfel $^{3}$ and Hiroki Sayama ${ }^{4}$ \\ ${ }^{1}$ Universidad Nacional Autónoma de México, Mexico City, Mexico \\ ${ }^{2}$ Institute of Cognitive Sciences and Technologies, Italian National Research Council, Rome, Italy \\ ${ }^{3}$ Wyss Institute for Biologically Inspired Enginering, Harvard University, Cambridge, MA 02138, USA \\ ${ }^{4}$ Binghamton University, Binghamton, NY 13902, USA \\ cgg@unam.mx, vito.trianni@istc.cnr.it, justin.werfel@wyss.harvard.edu, sayama@binghamton.edu
}

\begin{abstract}
Self-organization has been an important concept within a number of disciplines, which Artificial Life (ALife) also has heavily utilized since its inception. The term and its implications, however, are often confusing or misinterpreted. In this work, we provide a mini-review of self-organization and its relationship with ALife, aiming at initiating discussions on this important topic with the interested audience. We first articulate some fundamental aspects of self-organization, outline its usage, and review its applications to ALife within its soft, hard, and wet domains. We also provide perspectives for further research.
\end{abstract}

\section{What is self-organization?}

The term "self-organizing system" was coined by Ashby (1947) to describe phenomena where local interactions between independent elements lead to global behaviors or patterns. The phrase is used when an external observer perceives a pattern in a system with many components, and this pattern is not imposed by a central authority external to those components, but rather arises from the collective behavior of the elements themselves. Natural examples are found in areas such as collective motion (Vicsek and Zafeiris, 2012), as when birds or fish move in flocks or schools exhibiting complex group behavior; morphogenesis (Doursat, 2011), in which cells in a living body divide and specialize to develop into a complex body plan; and pattern formation (Cross and Hohenberg, 1993) in a variety of physical and chemical systems, such as convection and crystal growth as well as the formation of patterns like stripes and spots on animal coats.

A formal definition of the term runs into difficulties in agreeing on what is a system, what is organization, and what is self (Gershenson and Heylighen, 2003), none of which are perfectly straightforward. However, a pragmatic approach focuses on when it is useful to describe a system as self-organizing (Gershenson, 2007). This utility typically comes when an observer identifies a pattern at a higher scale but is also interested in phenomena at a lower scale; there then arise questions of how the lower scale produces the observables at the higher scale, as well as how the higher scale constrains and promotes observables at the lower scale. For example, bird behavior leads to flock formation, and descriptors at the level of the flock can also be used to understand regulation of individual bird behavior (Keys and Dugatkin, 1990).

Self-organization has been an important concept within a number of disciplines, such as statistical mechanics (Wolfram, 1983; Crutchfield, 2012), supramolecular chemistry (Lehn, 2017), and computer science (Mamei et al., 2006). Artificial Life (ALife) frequently draws heavily on self-organizing systems in different contexts Aguilar et al., 2014), starting in the early days of the field with studies of systems like snowflake formation (Packard, 1986) and agent flocking (Reynolds, 1987), and continuing to the present day. However, there are often confusion and misinterpretation involved with this concept, possibly due to an apparent lack of recent systematic literature. In this work, we aim at providing a mini-review of self-organization within the context of ALife, with a goal to open discussions on this important topic to the interested audience within the community. We first articulate some fundamental aspects of self-organization, outline ways the term has been used by researchers in the field, and then summarize work based on self-organization within soft (simulated), hard (robotic), and wet (chemical and biochemical) domains of ALife. We also provide perspectives of further research.

\section{Usage}

Ashby coined the term "self-organizing system" to show that a machine could be strictly deterministic and yet exhibit a self-induced change of organization (Ashby, 1947). This notion was further developed within cybernetics (von Foerster, 1960; Ashby, 1962). In many contexts, a thermodynamical perspective has been taken, where "organization" is viewed as the opposite of entropy (Nicolis and Prigogine, 1977). Since there is an equivalence between BoltzmannGibbs entropy and Shannon information, this notion has also been applied in contexts related to information theory (Fernández et al., 2014). In this view, a self-organizing system is one whose dynamics lead it to decrease its entropy 
or increase its information content. In the meantime, there are several other definitions of self-organization as well. For example, Shalizi (2001) defines self-organization as an increase in statistical complexity, which in turn is defined as the amount of information required to minimally specify the state of the system's causal architecture. As an alternative to entropy, the use of the mean value of random variables has also been proposed (Holzer and De Meer, 2011).

The recent subfield of guided self-organization explores mechanisms by which self-organization can be regulated for specific purposes-that is, how to find or design dynamics for a system such that it will have particular attractors or outcomes (Prokopenko, 2009; Av et al., 2012; Polani et al., 2013; Prokopenko, 2014; Prokopenko and Gershenson, 2014). Much of this research is based on information theory. For example, the self-organization of random Boolean networks (Kauffman, 1969, 1993) can be guided to specific dynamical regimes (Gershenson, 2012). The concept of self-organization is also heavily used in organization science, with relevance to artificial society models (Gilbert and Conte, 1995; Epstein and Axtell, 1996).

While there may be no single agreed-on definition of selforganization, this lack need not be an insurmountable obstacle for its study, any more than a lack of a unanimous formal definition of "life" has been an obstacle for progress in the fields of biology or ALife. In what follows, we provide a concice review of how self-organization has contributed to the progress of ALife.

\section{Domains}

One way to classify ALife research is to divide it into soft, hard, and wet domains, roughly referring to computer simulations, physical robots, and chemical/biological research (including living technology as the application of ALife (Bedau et al., 2009)), respectively. Self-organization has played a central role in work in all three domains.

\section{Soft ALife}

Soft ALife, or mathematical and computational modeling and simulation of life-like behaviors, has been linked to self-organization in many sub-domains. Cellular automata (CAs) (Ilachinski, 2001), one of the most popular modeling frameworks used in earlier forms of soft ALife, are well-explored, illustrative examples of self-organizing systems. A CA consists of many units (cells), each of which can be in any of a number of discrete states, and each of which repeatedly determines its next state in a fully distributed manner, based on its current state and those of its neighbors. With no central controller involved, CAs can spontaneously organize their state configurations to demonstrate various forms of self-organization: dynamical critical states such as in sand-pile models (Bak et al., 1988) and in the Game of Life (Bak et al., 1989), spontaneous formation of spatial patterns (Young, 1984; Wolfram, 1984; Ermentrout and Edelstein-Keshet, 1993), self-replication 11 (Langton, 1984, 1986; Reggia et al., 1993; Sipper, 1998), and evolution by variation and natural selection (Savama, 1999, 2004; Salzberg and Savama, 2004; Suzuki and Ikegami, 2006; Oros and Nehaniv, 2007, 2009). Similarly, partial differential equations (PDEs), a continuous counterpart of CAs, have an even longer history of demonstrating self-organizing dynamics (Turing, 1952; Glansdorff and Prigogine, 1971; Field and Noyes, 1974; Pearson, 1993).

Another representative class of soft ALife that shows self-organization comprises models of collective behavior of self-driven agents (Vicsek and Zafeiris, 2012). Reynolds' Boids model (Reynolds, 1987) is probably the best known in this category. In this work, self-propelled agents ("boids") move in a continuous space according to three kinetic rules: cohesion (to maintain positional proximity), alignment (to maintain directional similarity), and separation (to avoid overcrowding and collision). A variety of related models have since been proposed and studied, including simplified, statistical-physicsoriented ones (Vicsek et al., 1995; Levine et al., 2000; Aldana et al., 2007; Newman and Sayama, 2008) and more detailed, behavioral-ecology-oriented ones (Couzin et al., 2002; Kunz and Hemelrijk, 2003; Hildenbrandt et al., 2010). These models produce natural-looking flocking/schooling/swarming collective behaviors out of simple decentralized behavioral rules, and they also exhibit phase transitions between distinct macroscopic states.

Such collective behavior models have been brought to artificial chemistry studies (Dittrich et al., 2001; Banzhaf and Yamamoto, 2015) as well, such as swarm chemistry and its variants (Sayama, 2008; Kreyssig and Dittrich, 2011; Sayama, 2011, 2012; Erskine and Herrmann, 2015), in which kinetically and chemically distinct species of idealized agents interact to form nontrivial spatiotemporal dynamic patterns. More recently, these collective behavior models have also been actively utilized in morphogenetic engineering (Doursat, 2011; Doursat et al., 2012), in which researchers attempt to achieve a successful merger of self-organization and programmable architectural design, by discovering or designing agent rules that result in specific desired high-level patterns.

Other examples of self-organization in soft ALife are found in simulation models of artificial societies. Their roots can be traced back to the famous segregation mod-

\footnotetext{
${ }^{1}$ Note that earlier literature on self-reproducing cellular automata von Neumann, 1966; Codd, 1968) is not included here, because those models typically had a clear separation between a central universal controller and a structure that is procedurally constructed by the controller; thus they may not constitute a good example of self-organization as discussed in this article.
} 
els developed by Sakoda and Schelling back in the early 1970s (Sakoda, 1971; Schelling, 1971; Hegselmann, 2017), in which simple, independent decision making by individual agents would eventually cause a spatially segregated state of society at a macroscopic level. Agent-based simulation of artificial societies has been one of the core topics discussed in the ALife community (Epstein et al., 1996; Lansing, 2002), and has elucidated self-organization of issues in social order such as geographical resource management (Lansing and Kremer, 1993; Bousquet and Page, 2004), cooperative strategies (Lindgren and Nordahl, 1993; Brede, 2011; Adami et al., 2016; Ichinose and Savama, 2017), and common languages (Steels, 1995; Kirby, 2002; Smith et al., 2003; Lipowska and Lipowski, 2012). The literature on self-organization of adaptive social network structure (Gross and Savama, 2009; Bryden et al., 2010; Geard and Bullock, 2010) may also be included in this category.

\section{Hard ALife}

Robots can be considered to be life-like artifacts in their ability to sense their physical environment and take action in response. Physical agents, even very simple ones, can evoke in the observer a particularly strong sense of being animate. From W. Grey Walter's tortoises Walter, 1950, 1951), to simple machines based on the principles of Braitenberg's vehicles (Braitenberg, 1986), to other reactive robots (Brooks, 1989), to recent biomimetic and bioinspired designs (Saranli et al., 2001; Wood et al., 2013; Kim and Wensing, 2017), building artificial life as physically embodied hardware allows it to exploit the rich dynamics underlying the interaction between itself and its environment, so that even simple mechanisms and behavioral rules can confer sophisticated life-like attributes to limited machines (Simon, 1969). Still higher complexity can be attained either by increasing the sophistication of a single robot, or by increasing the number of robots in a system that, through the resulting interaction and self-organization, can then evince more sophisticated abilities collectively, from adaptive responses to group decision making.

Physical hardware has the strong advantage that the physical characteristics of the system (dynamics, sensor performance, actuator noise profiles, etc.) are by definition realistic, whereas simulations are necessarily simplified and typically fail to capture phenomena that only become evident through material experimentation Brooks and Matarić, 1993; Rubenstein et al., 2014). Conversely, while simulation can readily handle very large numbers of agents, hardware considerations (cost, space, scalability of operation, etc.) have traditionally limited hard ALife studies to using a small number of robots. In some scenarios, self-organizing phenomena of interest do not necessarily require a large number of robots; when the mechanism for coordination is based on stigmergy (persistent information left in a shared environment), the important element is a large number of interactions between robot and environment, and even a single robot could suffice (Beckers et al., 2000; Werfel et al., 2014). More recently, hardware advances have made it possible to conduct physical experiments with robots in numbers exceeding a thousand (Rubenstein et al., 2014).

Physical experiments have been used to explore selforganizing phenomena in a variety of areas. Aggregation of objects has been studied from a physics perspective (Giomi et al., 2013); in ways inspired by behavior observed in living systems, such as cockroaches or bees (Garnier et al., 2008; Kernbach et al., 2009); and using controllers designed through automatic methods like artificial evolution (Dorigo et al., 2004; Francesca et al., 2014). Another topic is collective navigation, in which groups of robots coordinate their overall direction of motion and collectively avoid obstacles (Baldassarre et al., 2007; Trianni and Dorigo, 2006; Turgut et al., 2008). In other collective decision-making processes, positive feedback from recruitment processes and negative feedback from cross-inhibition contribute to shape the outcome (Reina et al., 2018; Valentini et al., 2015; Scheidler et al., 2016; Garnier et al., 2009, 2013; Kernbach et al., 2009; Francesca et al., 2014; Valentini et al.,2017). Self-assembly (Whitesides and Grzybowski, 2002) is another form of selforganization, with several examples in hard ALife of self-assembling or self-reconfiguring robots (Murata et al., 1994; Holland and Melhuish, 1999; Støv and Nagpal, 2004; Zykov et al., 2005; Dorigo et al., 2006; Ampatzis et al., 2009; Rubenstein et al., 2014).

\section{Wet ALife}

Wet ALife, or physico-chemical synthesis of life-like behaviors, extensively utilizes self-organization as its core principle. A classic example is the spatial pattern formation in experimentally realized reaction-diffusion systems, such as the Belousov-Zhabotinsky reaction Vanag and Epstein, 2001; Adamatzky et al., 2008) and Gray-Scott-like selfreplicating spots (Lee et al., 1994), where dynamic patterns self-organize entirely from spatially localized chemical reactions. Similar approaches can also be taken by using microscopic biological organisms (e.g., slime molds) as the media of self-organization (Adamatzky et al., 2008; Adamatzky, 2015). In the origins of life research, molecular self-assembly plays the essential role in producing protocell structures and their metabolic dynamics (Rasmussen et al., 2003; Hanczyc et al., 2003; Rasmussen et al., 2004, 2008).

More recently, dynamic behaviors of macroscopically visible chemical droplets, a.k.a. liquid robots (Čejková et al., 2017), has become a focus of active research in ALife. In this line of research, interactions among chemical reactions, physical micro-fluid dynamics and possibly other not-yet-fully-understood microscopic mechanisms cause self-organization of spontaneous movements 
(Hanczyc et al., 2007; Ceikova et al., 2014) and complex morphology (Čejková et al., 2018) of those droplets. Moreover, droplet-based systems have also been used to demonstrate artificial evolution in experimental chemical systems (Parrilla-Gutierrez et al., 2017).

\section{Perspectives}

As already mentioned above, we can understand a selforganizing system as one in which organization increases in time. However, it can be shown that, depending on how the variables of a system are chosen, the same system can be said to be either organizing or disorganizing (Gershenson and Heylighen, 2003). Moreover, in several examples of self-organization, it is not straightforward to identify the self of the system, as oftentimes all elements composing the system can be ascribed equal agency. Finally, in cybernetics and systems theory, the dependency of the boundaries of a system on the observer has thoroughly been discussed (Gershenson et al., 2014): one wants to have an objective description of phenomena, but descriptions are necessarily made by observers, making them partially subjective. It becomes clear, then, that discussing selforganization requires the identification of what is self and what is other, and what are the elements that are increasing in their organization. Similar issues have been tackled by Maturana and Varela (1980) in the definition of living systems as autopoietic systems. According to this tradition, a living system is inherently self-organising because the self is continuously produced or renewed by processes brought forth by the system's internal components. In other words, an autopoietic system can be recognized as a unity with boundaries that encompass a number of simpler/elementary components that are at the basis of the organization of the system, as they are responsible for the definition of the system boundaries and for the (re)production of the very same components (Varela et al., 1974). This is a peculiar characteristic of living systems. If life is deeply rooted in selforganization, so should be ALife, and the several acceptations of ALife discussed above demonstrate the richness of the links it holds with self-organization.

Looking at the perspectives of ALife, it can be useful to think of self-organization as the common language that unifies the soft, hard and wet domains. The ALife community can progress owing to shared concepts and definitions, and despite the mentioned difficulties, self-organization stands as a common ground on which to build shared consensus. Most importantly, we believe that the identification and classifications of the mechanisms that underpin self-organization can be extremely useful to synthesize novel forms of ALife and gain a better understanding of life itself. These mechanisms should be identified at the level of the system components and characterized for the effects they have on the system organization. Mechanisms pertain to the modalities of interaction among system components (e.g., colli- sions, perceptions, direct communication, stigmergy), to behavioural patterns pertaining to individual components (e.g., exploration vs. exploitation), and to information enhancement or suppression (e.g., recruitment or inhibitory processes). The effects of the mechanisms should be visible in the creation of feedback loops-positive or negativeat the system level, which determine the complex dynamics underlying self-organization. We believe that, by identifying and characterising the mechanisms that support selforganization, the synthesis of artifacts with life-like properties would be much simplified. In this perspective, mechanisms underlying self-organization can be thought of as design patterns to generate artificial lives (Babaoglu et al., 2006; Fernandez-Marquez et al., 2013; Reina et al., 2014). By exploiting and composing them, different forms of ALife could be designed with a principled approach, owing to the understanding of the relationship between mechanisms and system organization.

The possibility of exploiting self-organization for design purposes is especially relevant toward the development of living technologies, that is, technologies presenting features of living systems (Bedau et al., 2009), such as robustness, adaptability, and self-organization, which can include self-reconfiguration, self-healing, selfmanagement, self-assembly, etc., often named together as "self-*" in the context of autonomic computing (Poslad, 2009). Self-organization has been used directly in living technologies within a variety of domains (Bedau et al., 2013), from protocells (Rasmussen et al., 2008) to cities (Gershenson, 2013), and also several methodologies that use self-organization have been proposed in engineering (Frei and Di Marzo Serugendo, 2011). A major leap forward can be expected when principled design methodologies are laid down, and a better understanding of selforganization for ALife can be at the forefront of the development of such methods.

As a final discussion, and as food for thought, it is also worth considering when self-organization is not useful in the context of ALife. Tracing a clear line across the domain is of course impossible, but our reasoning above provides some suggestions. Indeed, self-organization does not account for every life-like process, for instance when there is no clear increase in organization. For instance, hard ALife has strongly developed the concept of embodied cognition and morphological computation (Pfeifer and Gómez, 2009), where the dynamics of mind-body-environment interaction are fundamental aspects. These dynamics, albeit very complex, are not easily described within the framework of self-organization. Evolution is also very much represented within ALife, but typical generational evolutionary algorithms do not present clear elements of self-organization, as the progression in organization through generations is mediated by a central authority that defines selection of the fittest individuals. Open-ended evolution (Taylor et al., 
2016) makes a difference when such a centralized authority does not exist and progress is observable in mixing populations of interacting individuals. Exploring possibilities of infusing self-organization into those processes that were traditionally not oriented toward self-organization would be potentially a very fruitful direction of research.

Needless to say, this mini-review we presented here is not meant to be a complete, comprehensive review of selforganization and ALife, given the limitation of space. We plan to expand the work substantially in the near future to develop a more thorough review for publication elsewhere.

\section{References}

Adamatzky, A. (2015). A would-be nervous system made from a slime mold. Artificial life, 21(1):73-91.

Adamatzky, A., de Lacy Costello, B., and Shirakawa, T. (2008). Universal computation with limited resources: Belousovzhabotinsky and physarum computers. International Journal of Bifurcation and Chaos, 18(08):2373-2389.

Adami, C., Schossau, J., and Hintze, A. (2016). Evolutionary game theory using agent-based methods. Phys. Life Rev., 19:1-26.

Aguilar, W., Santamaría Bonfil, G., Froese, T., and Gershenson, C. (2014). The past, present, and future of artificial life. Frontiers in Robotics and AI, 1(8).

Aldana, M., Dossetti, V., Huepe, C., Kenkre, V. M., and Larralde, H. (2007). Phase transitions in systems of self-propelled agents and related network models. Physical Review Letters, 98:095702.

Ampatzis, C., Tuci, E., Trianni, V., Christensen, A., and Dorigo, M. (2009). Evolving self-assembly in autonomous homogeneous robots: experiments with two physical robots. Artificial Life.

Ashby, W. R. (1947). Principles of the self-organizing dynamic system. Journal of General Psychology, 37:125-128.

Ashby, W. R. (1962). Principles of the self-organizing system. In Foerster, H. V. and Zopf, Jr., G. W., editors, Principles of Self-Organization, pages 255-278, Oxford. Pergamon.

Ay, N., Der, R., and Prokopenko, M. (2012). Guided selforganization: perception-action loops of embodied systems. Theory in Biosciences, 131(3):125-127.

Babaoglu, O., Canright, G., Deutsch, A., Di Caro, G. A., Ducatelle, F., Gambardella, L. M., Ganguly, N., Jelasity, M. . r., Montemanni, R., Montresor, A., and Urnes, T. (2006). Design patterns from biology for distributed computing. ACM Transactions on Autonomous Adaptive Systems, 1(1):26-66.

Bak, P., Chen, K., and Kreutz, M. (1989). Self-organized criticality in the "game of life". Nature, 342:780-782.

Bak, P., Tang, C., and Wiesenfeld, K. (1988). Self-organized criticality. Physical Review A, 38:364.

Baldassarre, G., Trianni, V., Bonani, M., Mondada, F., Dorigo, M., and Nolfi, S. (2007). Self-organized coordinated motion in groups of physically connected robots. IEEE Trans. Syst. Man Cybern. B Cybern., 37:224-239.
Banzhaf, W. and Yamamoto, L. (2015). Artificial Chemistries. MIT Press.

Beckers, R., Holland, O. E., and Deneubourg, J.-L. (2000). Prerational Intelligence: Adaptive Behavior and Intelligent Systems Without Symbols and Logic, Volume 2, chapter From Local Actions to Global Tasks: Stigmergy and Collective Robotics. Springer, Dordrecht.

Bedau, M. A., McCaskill, J. S., Packard, N. H., Parke, E. C., and Rasmussen, S. R. (2013). Introduction to recent developments in living technology. Artificial Life, 19(3):291-298.

Bedau, M. A., McCaskill, J. S., Packard, N. H., and Rasmussen, S. (2009). Living technology: Exploiting life's principles in technology. Artificial Life, 16(1):89-97.

Bousquet, F. and Page, C. L. (2004). Multi-agent simulations and ecosystem management: a review. Ecological Modelling, 176(3-4):313 - 332 .

Braitenberg, V. (1986). Vehicles: Experiments in synthetic psychology. MIT Press, Cambridge, MA, USA.

Brede, M. (2011). The evolution of cooperation on correlated payoff landscapes. Artificial Life, 17(4):365-373.

Brooks, R. A. (1989). A robot that walks; emergent behaviors from a carefully evolved network. In IEEE International Conference on Robotics and Automation.

Brooks, R. A. and Matarić, M. J. (1993). Robot Learning, chapter Real Robots, Real Learning Problems, pages 193-213. Kluwer Academic Press.

Bryden, J., Funk, S., Geard, N., Bullock, S., and Jansen, V. A. A. (2010). Stability in flux: community structure in dynamic networks. J. Roy. Soc. Interface, 8(60):1031-1040.

Čejková, J., Banno, T., Hanczyc, M. M., and Štěpánek, F. (2017). Droplets as liquid robots. Artificial Life, 23(4):528-549.

Čejková, J., Hanczyc, M. M., and Štěpánek, F. (2018). Multiarmed droplets as shape-changing protocells. Artificial Life, 24(1):71-79.

Cejkova, J., Novak, M., Stepanek, F., and Hanczyc, M. M. (2014). Dynamics of chemotactic droplets in salt concentration gradients. Langmuir, 30(40):11937-11944.

Codd, E. F. (1968). Cellular Automata, chapter A SelfReproducing Universal Computer-Constructor, pages 81105. Academic Press, Inc.

Couzin, I. D., Krause, J., James, R., Ruxton, G. D., and Franks, N. R. (2002). Collective memory and spatial sorting in animal groups. Journal of Theoretical Biology, 218(1):1-11.

Cross, M. C. and Hohenberg, P. C. (1993). Pattern formation outside of equilibrium. Rev. Mod. Phys., 65:851-1112.

Crutchfield, J. P. (2012). Between order and chaos. Nature Physics, $8: 17-24$.

Dittrich, P., Ziegler, J., and Banzhaf, W. (2001). Artificial chemistries - a review. Artificial Life, 7(3):225-275. 
Dorigo, M., Trianni, V., Şahin, E., Groß, R., Labella, T. H., Baldassarre, G., Nolfi, S., Deneubourg, J.-L., Mondada, F., Floreano, D., and Gambardella, L. (2004). Evolving selforganizing behaviors for a swarm-bot. Autonomous Robots, 17(2-3):223-245.

Dorigo, M., Tuci, E., Trianni, V., Groß, R., Nouyan, S., Ampatzis, C., Labella, T. H., O'Grady, R., Bonani, M., and Mondada, F. (2006). Computational Intelligence: Principles and Practice, chapter SWARM-BOT: Design and implementation of colonies of self-assembling robots. IEEE Computational Intelligence Society.

Doursat, R. (2011). The myriads of alife: Importing complex systems and self-organization into engineering. In 2011 IEEE Symposium on Artificial Life.

Doursat, R., Sayama, H., and Michel, O., editors (2012). Morphogenetic Engineering: Toward Programmable Complex Systems. Springer-Verlag, Berlin, Heidelberg.

Epstein, J., Axtell, R., and Project, . (1996). Growing Artificial Societies: Social Science from the Bottom Up. A Bradford book. Brookings Institution Press.

Epstein, J. M. and Axtell, R. L. (1996). Growing Artificial Societies: Social Science from the Bottom Up. Brookings Institution Press MIT Press.

Ermentrout, G. B. and Edelstein-Keshet, L. (1993). Cellular automata approaches to biological modeling. Journal of Theoretical Biology, 160(1):97-133.

Erskine, A. and Herrmann, J. M. (2015). Cell-division behavior in a heterogeneous swarm environment. Artif. Life, 4(481-500).

Fernández, N., Maldonado, C., and Gershenson, C. (2014). Information measures of complexity, emergence, selforganization, homeostasis, and autopoiesis. In Prokopenko, M., ed., Guided Self-Organization: Inception, pages 19-51.

Fernandez-Marquez, J. L., Di Marzo Serugendo, G., Montagna, S., Viroli, M., and Arcos, J. L. (2013). Description and composition of bio-inspired design patterns: a complete overview. Natural Computing, 12(1):43-67.

Field, R. J. and Noyes, R. M. (1974). Oscillations in chemical systems. IV. Limit cycle behavior in a model of a real chemical reaction. The Journal of Chemical Physics, 60(5).

Francesca, G., Brambilla, M., Brutschy, A., Trianni, V., and Birattari, M. (2014). Automode: A novel approach to the automatic design of control software for robot swarms. Swarm Intelligence, 8(2):89-112.

Frei, R. and Di Marzo Serugendo, G. (2011). Advances in complexity engineering. Int. J. of Bio-Inspired Computation, 3(4):199-212.

Garnier, S., Combe, M., Jost, C., and Theraulaz, G. (2013). Do ants need to estimate the geometrical properties of trail bifurcations to find an efficient route? a swarm robotics test bed. PLOS Computational Biology, 9:e1002903.

Garnier, S., Gautrais, J., Asadpour, M., Jost, C., and Theraulaz, G. (2009). Self-organized aggregation triggers collective decision making in a group of cockroach-like robots. Adaptive Behavior, 17(2):109-133.
Garnier, S., Jost, C., Gautrais, J., Asadpour, M., Caprari, G., Jeanson, R., Grimal, A., and Theraulaz, G. (2008). The embodiment of cockroach aggregation behavior in a group of microrobots. Artificial Life, 14(4):387-408.

Geard, N. and Bullock, S. (2010). Competition and the dynamics of group affiliation. Advances in Complex Systems, 13(4):501.

Gershenson, C. (2007). Design and Control of Selforganizing Systems. CopIt Arxives, Mexico. http://tinyurl.com/DCSOS2007.

Gershenson, C. (2012). Guiding the self-organization of random Boolean networks. Theory in Biosciences, 131(3):181-191.

Gershenson, C. (2013). Living in living cities. Artificial Life, 19(3 \& 4):401-420.

Gershenson, C., Csermely, P., Erdi, P., Knyazeva, H., and Laszlo, A. (2014). The past, present and future of cybernetics and systems research. systema: connecting matter, life, culture and technology, 1(3):4-13.

Gershenson, C. and Heylighen, F. (2003). When can we call a system self-organizing? In ECAL 2003, pages 606-614.

Gilbert, N. and Conte, R., editors (1995). Artificial Societies: the computer simulation of social life. Taylor \& Francis, Inc., Bristol, PA, USA.

Giomi, L., Hawley-Weld, N., and Mahadevan, L. (2013). Swarming, swirling and stasis in sequestered bristle-bots. Proc. $R$. Soc. A, 469(2151).

Glansdorff, P. and Prigogine, I. (1971). Thermodynamic Theory of Structure, Stability and Fluctuations. Wiley-Interscience, New York.

Gross, T. and Sayama, H., editors (2009). Adaptive networks: Theory, Models and Applications. Understanding Complex Systems. Springer, Berlin Heidelberg.

Hanczyc, M. M., Fujikawa, S. M., and Szostak, J. W. (2003). Experimental models of primitive cellular compartments: encapsulation, growth, and division. Science, 302:618-622.

Hanczyc, M. M., Toyota, T., Ikegami, T., Packard, N., and Sugawara, T. (2007). Fatty acid chemistry at the oil- water interface: self-propelled oil droplets. Journal of the American Chemical Society, 129(30):9386-9391.

Hegselmann, R. (2017). Thomas C. Schelling and James M. Sakoda: The intellectual, technical, and social history of a model. J. Artif. Soc. Soc. Simul., 20(3):15.

Hildenbrandt, H., Carere, C., and Hemelrijk, C. (2010). Selforganized aerial displays of thousands of starlings: a model. Behavioral Ecology, 21(6):1349-1359.

Holland, O. and Melhuish, C. (1999). Stigmergy, self-organization, and sorting in collective robotics. Artificial Life, 5:173-202.

Holzer, R. and De Meer, H. (2011). Methods for approximations of quantitative measures in self-organizing systems. In Bettstetter, C. and Gershenson, C., editors, Self-Organizing Systems, $L C N S$ volume 6557 , pages $1-15$. 
Ichinose, G. and Sayama, H. (2017). Invasion of cooperation in scale-free networks: Accumulated versus average payoffs. Artificial Life, 23(1):25-33.

Ilachinski, A. (2001). Cellular Automata: A Discrete Universe. World Scientific.

Kauffman, S. A. (1969). Metabolic stability and epigenesis in randomly constructed genetic nets. J. Theor. Biol., 22:437-467.

Kauffman, S. A. (1993). The Origins of Order. Oxford University Press, Oxford, UK.

Kernbach, S., Thenius, R., Kernbach, O., and Schmickl, T. (2009). Re-embodiment of honeybee aggregation behavior in an artificial micro-robotic system. Adapt. Behav., 17(3):237-259.

Keys, G. C. and Dugatkin, L. A. (1990). Flock size and position effects on vigilance, aggression, and prey capture in the european starling. The Condor, 92:151-159.

Kim, S. and Wensing, P. M. (2017). Foundations and Trends in Robotics, volume 5, chapter Design of Dynamic Legged Robots, pages 117-190. Now Publishers Inc.

Kirby, S. (2002). Natural language from artificial life. Artifical Life, 8(2):185 - 215.

Kreyssig, P. and Dittrich, P. (2011). Reaction flow artificial chemistries. In ECAL, pages 431-437.

Kunz, H. and Hemelrijk, C. K. (2003). Artificial fish schools: Collective effects of school size, body size, and body form. Artificial Life, 9(3):237-253.

Langton, C. G. (1984). Self-reproduction in cellular automata. Physica D, 10:135-144.

Langton, C. G. (1986). Studying artificial life with cellular automata. Physica D, 22(1-3):129-149.

Lansing, J. S. (2002). "artificial societies" and the social sciences. Artificial Life, 8(3):279-292.

Lansing, J. S. and Kremer, J. N. (1993). Emergent properties of balinese water temple networks: Coadaptation on a rugged fitness landscape. American Anthropologist, 95(1):97-114.

Lee, K.-J., McCormick, W. D., Pearson, J. E., and Swinney, H. L. (1994). Experimental observation of self-replicating spots in a reaction-diffusion system. Nature, 369(6477):215.

Lehn, J.-M. (2017). Supramolecular chemistry: Where from? where to? Chem. Soc. Rev., 46:2378-2379.

Levine, H., Rappel, W.-J., and Cohen, I. (2000). Self-organization in systems of self-propelled particles. Physical Review E, 63:017101.

Lindgren, K. and Nordahl, M. G. (1993). Cooperation and community structure in artificial ecosystems. Artificial Life, 1(1_2):15-37.

Lipowska, D. and Lipowski, A. (2012). Naming game on adaptive weighted networks. Artificial Life, 18(3):311-323.

Mamei, M., Menezes, R., Tolksdorf, R., and Zambonelli, F. (2006). Case studies for self-organization in computer science. Journal of Systems Architecture, 52(8-9):443-460.
Maturana, H. and Varela, F. (1980). Autopoiesis and Cognition: the Realization of the Living. D. Reidel Publishing Co., Dordecht, 2nd edition.

Murata, S., Kurokawa, H., and Kokaji, S. (1994). Self-assembling machine. In Proc. ICRA 1994.

Newman, J. P. and Sayama, H. (2008). Effect of sensory blind zones on milling behavior in a dynamic self-propelled particle model. Physical Review E, 78:011913.

Nicolis, G. and Prigogine, I. (1977). Self-Organization in NonEquilibrium Systems: From Dissipative Structures to Order Through Fluctuations. Wiley, Chichester.

Oros, N. and Nehaniv, C. L. (2007). Sexyloop: Self-reproduction, evolution and sex in cellular automata. In 2007 IEEE Symposium on Artificial Life.

Oros, N. and Nehaniv, C. L. (2009). Dude, where is my sex gene?-persistence of sex over evolutionary time in cellular automata. In 2009 IEEE Symposium on Artificial Life.

Packard, N. (1986). Lattice models for solidification and aggregation. In Theory and Application of Cellular Automata, Wolfram $S$. (ed.), pages 305-310. World Scientific, Institute for Advanced Study Preprint. Reprinted (1986).

Parrilla-Gutierrez, J. M., Tsuda, S., Grizou, J., Taylor, J., Henson, A., and Cronin, L. (2017). Adaptive artificial evolution of droplet protocells in a 3d-printed fluidic chemorobotic platform with configurable environments. Nature Communications, 8(1): 1144

Pearson, J. E. (1993). Complex patterns in a simple system. Science, 261(5118):189-192.

Pfeifer, R. and Gómez, G. (2009). Morphological Computation - Connecting Brain, Body, and Environment. In Creating Brain-Like Intelligence, pages 66-83. Springer, Berlin.

Polani, D., Prokopenko, M., and Yaeger, L. S. (2013). Information and self-organization of behavior. Advances in Complex Systems, 16(2\&3):1303001.

Poslad, S. (2009). Autonomous Systems and Artificial Life, chapter 10, pages 317-341. Wiley-Blackwell.

Prokopenko, M. (2009). Guided self-organization. HFSP Journal, 3(5):287-289.

Prokopenko, M., editor (2014). Guided Self-Organization: Inception, volume 9 of Emergence, Complexity and Computation. Springer, Berlin Heidelberg.

Prokopenko, M. and Gershenson, C. (2014). Entropy methods in guided self-organisation. Entropy, 16(10):5232-5241.

Rasmussen, S., Bedau, M. A., Chen, L., Deamer, D., Krakauer, D. C., Packard, N. H., and Stadler, P. F., editors (2008). Protocells: Bridging Nonliving and Living Matter Bridging Nonliving and Living Matter. MIT Press.

Rasmussen, S., Chen, L., Deamer, D., Krakauer, D. C., Packard, N. H., Stadler, P. F., and Bedau, M. A. (2004). Transitions from nonliving to living matter. Science, 303(5660):963-965.

Rasmussen, S., Chen, L., Nilsson, M., and Abe, S. (2003). Bridging nonliving and living matter. Artificial life, 9(3):269-316. 
Reggia, J. A., Armentrout, S. L., Chou, H.-H., and Peng, Y. (1993). Simple systems that exhibit self-directed replication. Science, 259(5099):1282-1287.

Reina, A., Bose, T., Trianni, V., and Marshall, J. A. R. (2018). Effects of spatiality on value-sensitive decisions made by robot swarms. In DARS 2016, pages 461-473.

Reina, A., Dorigo, M., and Trianni, V. (2014). Towards a Cognitive Design Pattern for Collective Decision-Making. In ANTS'14, pages 194-205.

Reynolds, C. W. (1987). Flocks, herds, and schools: A distributed behavioral model. Computer Graphics, 21(4):25-34.

Rubenstein, M., Cornejo, A., and Nagpal, R. (2014). Programmable self-assembly in a thousand-robot swarm. Science, 345(6198):795-799.

Sakoda, J. M. (1971). The checkerboard model of social interaction. The Journal of Mathematical Sociology, 1(1):119-132.

Salzberg, C. and Sayama, H. (2004). Complex genetic evolution of artificial selfreplicators in cellular automata. Complexity, 10(2):33-39.

Saranli, U., Buehler, M., and Koditschek, D. E. (2001). RHex: A simple and highly mobile robot. International Journal of Robotics Research, 20(7):616-631.

Sayama, H. (1999). A new structurally dissolvable selfreproducing loop evolving in a simple cellular automata space. Artificial Life, 5(4):343-365.

Sayama, H. (2004). Self-protection and diversity in self-replicating cellular automata. Artificial Life, 10(1):83-98.

Sayama, H. (2008). Swarm chemistry. Artif. Life, 15(1):105-114.

Sayama, H. (2011). Seeking open-ended evolution in swarm chemistry. In 2011 IEEE Symposium on Artificial Life.

Sayama, H. (2012). Morphologies of self-organizing swarms in 3d swarm chemistry. In Proc. GECCO 2012, pages 577-584.

Scheidler, A., Brutschy, A., Ferrante, E., and Dorigo, M. (2016). The k-unanimity rule for self-organized decision-making in swarms of robots. IEEE Transactions on Cybernetics, 46(5):1175-1188

Schelling, T. C. (1971). Dynamic models of segregation. The Journal of Mathematical Sociology, 1(2):143-186.

Shalizi, C. R. (2001). Causal Architecture, Complexity and SelfOrganization in Time Series and Cellular Automata. PhD thesis, University of Wisconsin at Madison.

Simon, H. A. (1969). The Sciences of the Artificial. MIT Press, Cambridge, Massachusetts.

Sipper, M. (1998). Fifty years of research on self-replication: An overview. Artificial Life, 4(3):237-257.

Smith, K., Kirby, S., and Brighton, H. (2003). Iterated learning: A framework for the emergence of language. Artificial Life, 9(4):371-386.

Steels, L. (1995). A self-organizing spatial vocabulary. Artificial Life, 2(3):319-332.
Støy, K. and Nagpal, R. (2004). Self-reconfiguration using directed growth. In Proc. DARS 2004, Toulouse, France.

Suzuki, K. and Ikegami, T. (2006). Spatial-pattern-induced evolution of a self-replicating loop network. Artificial Life, 12(4):461-485

Taylor, T., et al. (2016). Open-ended evolution: perspectives from the OEE workshop in York. Artificial Life, 22(3):408-423.

Trianni, V. and Dorigo, M. (2006). Self-organisation and communication in groups of simulated and physical robots. Biological Cybernetics, 95(3):213-231.

Turgut, A. E., Çelikkanat, H., Gökçe, F., and Şahin, E. (2008). Self-organized flocking in mobile robot swarms. Swarm Intelligence, 2(2-4):97-120.

Turing, A. (1952). The chemical basis of morphogenesis. Phil. Trans. Roy. Soc. Lond. B, 237(641):37-72.

Valentini, G., Ferrante, E., and Dorigo, M. (2017). The best-of- $n$ problem in robot swarms: Formalization, state of the art, and novel perspectives. Frontiers in Robotics and AI, 4(9).

Valentini, G., Ferrante, E., Hamann, H., and Dorigo, M. (2015). Collective decision with 100 kilobots: speed versus accuracy in binary discrimination problems. In Autonomous Agents and Multi-Agent Systems, pages 553-580.

Vanag, V. K. and Epstein, I. R. (2001). Pattern formation in a tunable medium: The Belousov-Zhabotinsky reaction in an aerosol OT microemulsion. Phys. Rev. Lett., 87(22):228301.

Varela, F. J., Maturana, H. R., and Uribe, R. (1974). Autopoiesis: The organization of living systems, its characterization and a model. Biosystems, 5(4):187-196.

Vicsek, T., Czirók, A., Ben-Jacob, E., Cohen, I., and Shochet, O. (1995). Novel type of phase transition in a system of selfdriven particles. Physical Review Letters, 75:1226.

Vicsek, T. and Zafeiris, A. (2012). Collective motion. Physics Reports, 517:71-140.

von Foerster, H. (1960). On self-organizing systems and their environments. In Yovitts, M. C. and Cameron, S., editors, SelfOrganizing Systems, pages 31-50, New York. Pergamon.

von Neumann, J. (1966). The Theory of Self-Reproducing Automata. University of Illinois Press, Champaign. Edited by A. W. Burks.

Walter, W. G. (1950). An imitation of life. Sci. Am., 182:42-45.

Walter, W. G. (1951). A machine that learns. Sci. Am., 185:60-63.

Werfel, J., Petersen, K., and Nagpal, R. (2014). Designing collective behavior in a termite-inspired robot construction team. Science, 343(6172):754-758.

Whitesides, G. M. and Grzybowski, B. (2002). Self-assembly at all scales. Science, 295(5564):2418-2421.

Wolfram, S. (1983). Statistical mechanics of cellular automata. Reviews of Modern Physics, 55(3):601-644.

Wolfram, S. (1984). Cellular automata as models of complexity. Nature, 311:419-424. 
Wood, R., Nagpal, R., and Wei, G.-Y. (2013). Flight of the robobees. Scientific American.

Young, D. A. (1984). A local activator-inhibitor model of vertebrate skin patterns. Mathematical Biosciences, 72(1):51-58.

Zykov, V., Mytilinaios, E., Adams, B., and Lipson, H. (2005). Robotics: self-reproducing machines. Nature, 435:163-164. 